\title{
A Novel Frequency Locked Loop Based on Stochastic Resonance
}

\author{
Weitong Zhang \\ Postgraduate Team 2, ICE, PLAUST \\ Nanjing, China \\ 15605143455@163.com
}

\author{
Zhiqiang Li,Wenming Zhu \\ Department of space-based information system \\ ICE, PLAUST \\ Nanjing, China
}

\begin{abstract}
Frequency locked loop (FLL) plays an important role in carrier synchronization because of its excellent dynamic performance. However, it performs inadequately in low signalto-noise ratio (SNR). In this paper, the principle of stochastic resonance (SR) is briefly introduced and a SR processor is proposed. Based on traditional FLL, the SR processor is added before frequency discriminator in order to weaken the effect that thermal noise brings to FLL. The paper investigates the processing effect of SR. Simulation results show that the performance of improved FLL is greatly improved. It can tolerate rather high dynamics and tracking accuracy of frequency achieve $0.2 \mathrm{~Hz}$ even with CNR as low as $25 \mathrm{dBHz}$, which verified the validity of above ideas.
\end{abstract}

Keywords- stochastic resonance ; carrier tracking ; frequency-locked loop; low signal-to-noise ratio

\section{INTRODUCTION}

Carrier tracking is extremely important for a TT\&C (Telemetry, Tracking and Command) system. Frequency locked loop (FLL) is an essential part of it and the total carrier tracking error is mostly comprised of the thermal noise error and dynamic stress error. For frequency locked loop design, the principal strategy to improve the loop performance is loop adjusting bandwidth in the most instances. Narrow bandwidth can reduce the thermal noise error but increase the dynamic stress error, while wide bandwidth will improve the dynamic performance of the loop but increase the thermal noise error.

In order to improve the performance of FLL, people have made a great effort. The improved FLL structure in [1] is very complex because the discriminator makes use of two kinds of frequency estimation algorithms, and its identify results take the outliers eliminate technology, and its loop filter bandwidth switches following the system change. Though the performance of the FLL improves, it is very complex and not easy to be put into actual use.

Now the major problem is how to weaken the effect of noise. In the fields of traditional signal processing, noise is regarded as the main reason for the deterioration performance. From the viewpoint of SR, suitable noise does not deteriorate the nonlinear system any more, however, it might have some constructive effect on the performance of the system ${ }^{[2]}$. The basic principle of stochastic resonance is when the input signal matches with the noise in a nonlinear system, part of the noise energy will transfer to the signal energy and increase carrier to noise ratio greatly. At first, stochastic resonance can be used only in small value (the frequency is Less than $1 \mathrm{~Hz}$ ) signal processing. Leng ${ }^{[3]}$ have proposed a new scheme called twice sampling stochastic resonance to deal with big value signal(the frequency is Less than $50 \mathrm{~Hz}$ ). Considering that the frequency offset after carrier capturing is just in the scope, we can process the input signal with stochastic resonance to weaken the effect of noise.

Up to now, works on this aspect have rarely been seen in public references. According to this, a new method of FLL structure is proposed in this paper which is based on stochastic resonance. This paper is organized as follows: in section II, the basic theory of stochastic resonance is introduced simply. Improved FLL is discussed in section III. In section IV, the simulation results are given out. Finally, a brief summary is given and conclusion is drawn in last section.

\section{BASIC THEORY OF StOCHASTIC RESONANCE}

In 1981, R.Benzi ${ }^{[4]}$ first proposed the conception of stochastic resonance which was used to explain the periodicity in the earth's climate change for last 700,000 years. The cooperative phenomenon of nonlinear system, noise and weak signal is named as stochastic resonance. With the deepening of research, people found that SR is not particular phenomena of special systems, which have been observed largely from physical systems ${ }^{[5]}$ to biomedical systems ${ }^{[6]}$. SR has been widely applied in weak signal processing for its great improvement of signal to noise ratio (SNR).

The overdamped motion of a Brownian particle in the bistable system in the presence of noise and external force could be used to depict SR. The output of the bistable system satisfies the Langevin equation:

$$
\frac{d x(t)}{d t}=a x(t)-b[x(t)]^{3}+s(t)+n(t)
$$

In the equation, $s(t)$ is the input signal, and $x(t)$ is the output signal, and $n(t)$ is the Gaussian white noise whose autocorrelation function is $\langle n(t) n(t+\tau)\rangle=D \delta(\tau)$. D is the variance of noise, $a$ and $b$ are system parameters. The potential function of the bistable system is:

$$
V(x)=-\frac{a}{2} x^{2}+\frac{b}{4} x^{4}
$$

The potential function curve can be described in a double-well potential diagram shown in Fig. 1. 


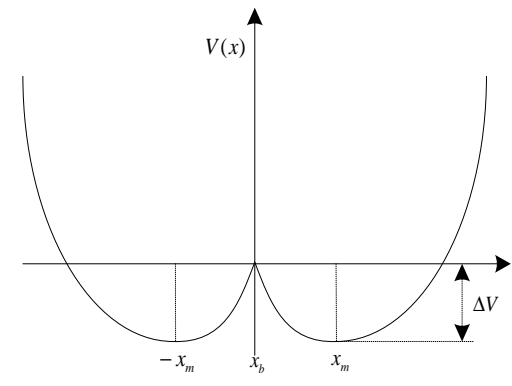

Figure 1. Potential function system

We can see that there are two minimal values of the potential function locating at $x= \pm x_{m}= \pm \sqrt{a / b}$ and a maximum value locating at $x_{b}=0$. The barrier height is $\Delta V=a^{2} / 4 b$. When both external periodic signal and the noise intensity $\sigma$ are zero, the particle will still in a potential well point. When only the noise exists, the particle in the noise-driven flips between two potential well. When external periodic signal exists, the height of the potential wells will be tuned by the weak external force.The change of $V(x)$ is showen in Fig. 2. We define $\Delta V_{\text {min }}$ as the absolute minimum difference of the potential well and the potential barrier when potential function changes. When the particle can leap over $\Delta V_{\min }$ aided by the noise, and the cycle of the particle is synchronized with the change of signal, stochastic resonance phenomenon occurs.

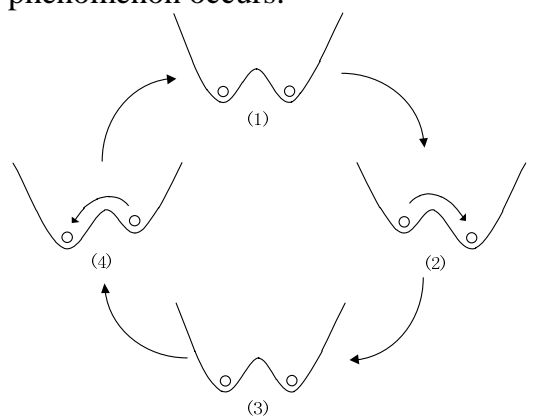

Figure 2. Change of potential function system

Tuning the intensity of noise $\mathrm{e}^{[7]}$ is the main method in original research on SR focused. In 1993, V.Anishchenko ${ }^{[8]}$ pointed out that a more universal theory of SR is tuning the nonlinear system under the noise of fixed intensity. $\mathrm{Xu}^{[9]}$ introduced the concept of response rate to explain the generation mechanism of SR in 2002. Duan ${ }^{[10]}$ testified the consistency between the method of tuning system parameter with that of tuning noise in 2003. Tuning parameter has an advantage than tuning the intensity of noise, which promotes the application of SR greatly.

We can do the replacement as follows.

$$
y(t)=\frac{x(t)}{\sigma}, \bar{b}=b \times \sigma^{2}, \bar{s}(t)=\frac{s(t)}{\sigma}
$$

when (3) is imported, (1) turns into:

$$
\frac{d y(t)}{d t}=a y(t)-\bar{b}[y(t)]^{3}+\bar{s}(t)+\xi(t)
$$

$\xi(t)$ in (4) is the unitary noise.

The potential function of (4) is :

$$
V[y(t)]=-\frac{1}{2} a[y(t)]^{2}+\frac{1}{4} \bar{b}[y(t)]^{4}
$$

The new potential barrier height is:

$$
\Delta V^{\prime}=\frac{a^{2}}{4 \bar{b}}=\frac{a^{2}}{4 b \sigma}
$$

From (6), we can see that tuning system parameter and tuning noise could both change the height of potential wells. Tuning system parameter can either strengthen the effect of nonlinear systems or weaken the effct of the nonlinear system. We all know that reducing noise is not an easy thing, so tuning system parameter appears very necessary.

Inspired by SR of tuning system parameter and its performance, we can firstly process the frequency offset to weaken the effect of noise. An improved FLL structure which is very flexible and effective is put forward.

\section{THE IMPROVED FLL STRUCTURE}

In traditional frequency locked loop, the signal which has been orthogonally demodulated directly will feed into the discriminator to get the frequency offset. Because of the effects of noise, the result of the discriminator is unsatisfactory. In order to get high tracking precision with the frequency locked loop in high dynamic circumstance and low carrier to noise ratio, a stochastic resonance pretreatment processor is added before the discriminator. The improved FLL structure is shown in Fig. 3.

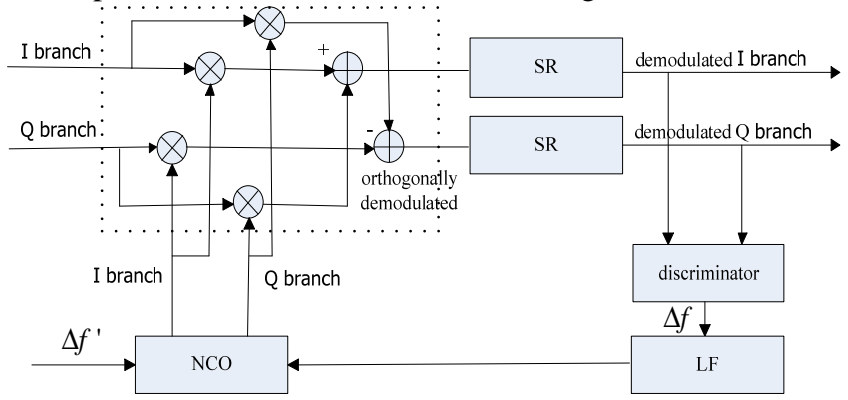

Figure 3. The improved FLL structure

$\Delta f$ ' in Fig. 3 is the coarse value of the carrier frequency offset provided by the carrier capture processor. $I_{p s}$ is the inphase branch and $Q_{p s}$ is the quadrature-phase branch. After orthogonal demodulation, we can get the instant frequency offset. With the procession of stochastic resonance, the negative effect of the noise is weakened. $\Delta f$ is calculated with the frequency discriminator. Through the procession of the loop filter, control gain is obtained and then it will adjust the NCO in order to generate a new frequency. Then, a closed feedback frequency locked loop is formed. After continuously cycle, local carrier generator will generate the same frequency as the carrier.

According to (1), the SR processor constructed in the Simulink environment as follows. There are two accumulation modules and an integration module. $a$ and 
$b$ are system parameters. The troublesome problem for SR processor is how to choose the best value of system parameters, which has not been well solved yet. Generally, the system parameters are determined by experience in practice. Now some methods of blind self-adaptive stochastic resonance have been proposed. In the paper we adjust $a$ and $b$ by experiences until stochastic resonance occur.

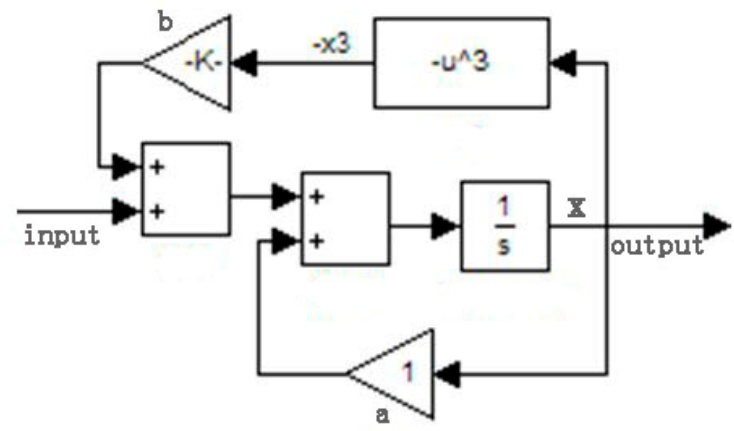

Figure 4. The SR processor

Another important thing to FLL is the discriminator algorithm. Considering arctangent algorithm is better than other discriminator algorithms in the high and low SNR, and independent of the slope and the signal amplitude, FLL adopts arctangent discriminator algorithm ${ }^{[11]}$. The arctangent cross product frequency discriminator output is:

$$
\begin{aligned}
\omega(k) & =\operatorname{actan}\left(\frac{I_{p s 1} \cdot Q_{p s 2}-I_{p s 2} \cdot Q_{p s 1}}{I_{p s 1} \cdot I_{p s 2}-Q_{p s 2} \cdot Q_{p s 1}}\right) \\
& =\varphi(k)-\varphi(k-1)+n
\end{aligned}
$$

As for loop bandwidth, it is very difficult to choose. Just as described in section I, there is a tradeoff involvement between the two opposing consideration. In order to make the effect of stochastic resonance more apparent, a wide loop bandwidth is adopted in this paper.

\section{SIMULATION AND RESUlT}

To check the performance of the improved FLL structure, a computer simulation is carried out. The simulation condition is designed for the weak signal between navigation satellite and Ground station. The carrier signal is implemented as sinusoidal periodic signal buried with additive Gaussian white noise. The frequency offset is not more than $20 \mathrm{~Hz}$ after capturing. A wide loop bandwidth is adopted in this paper. All in all, we set the parameters as follows:

Signal carrier to noise ratio: $25 \mathrm{dBHz}$

Doppler frequency rate: $5 \mathrm{~Hz} / \mathrm{s}$

Loop coherent integration time : $5 \mathrm{~ms}$

Loop bandwidth: $2 \mathrm{~Hz}$

According to the structure in Fig. 3, we implement the simulation based on the MATLAB environment with the above parameters.
A mass of researches have indicated that the parameter $a$ has little effect on the performance of the system. We set $a=1$, and make the nonlinear system to access stochastic resonance state by adjusting the parameters of $b$.According to the experience, We firstly set $b=1000000$.

Fig. 5 shows that the carrier signal is affected by the noise and the shape of the wave jitters severely. The content in Fig. 6 is the result of the processing of stochastic resonance with the parameter $a=1$ and $b=100000000$.

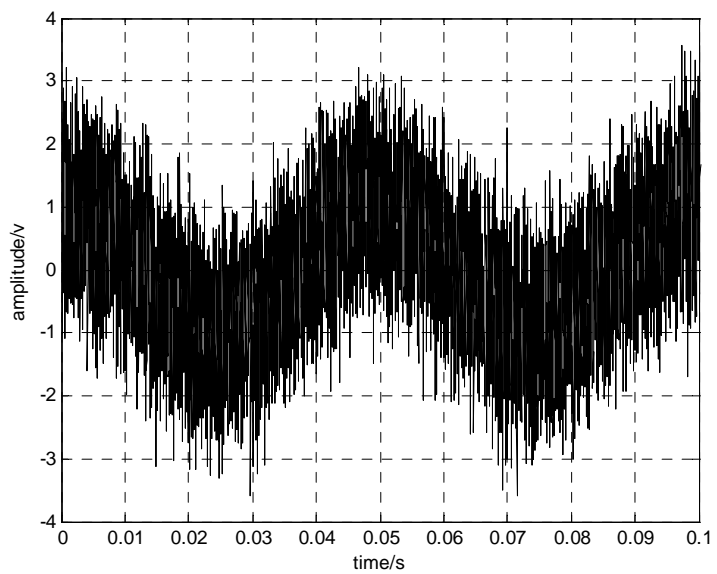

Figure 5. Carrier without processing of stochastic resonance

Through the nonlinear effect of stochastic resonance, the signal strength drops nearly 3 orders of magnitude. At the same time, the influence of the noise has been greatly weakened and the waveform of the signal becomes more smooth and stable. We can also find that the waveform has a slightly delay which does not affect the tracking of FLL. In this way, the noise entering FLL is reduced, and high-precision frequency tracking of FLL can be achieved.

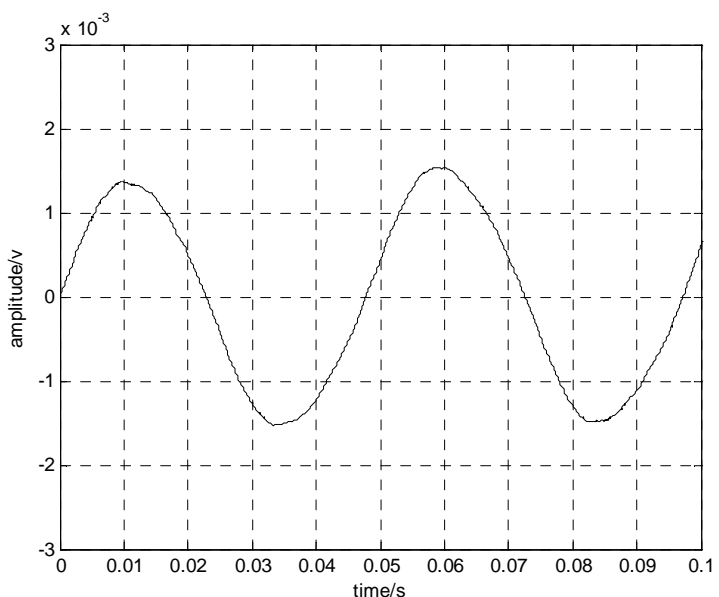

Figure 6. Carrier with processing of stochastic resonance

The frequency tracking performance of traditional FLL and improved FLL are shown in the following, respectively. 


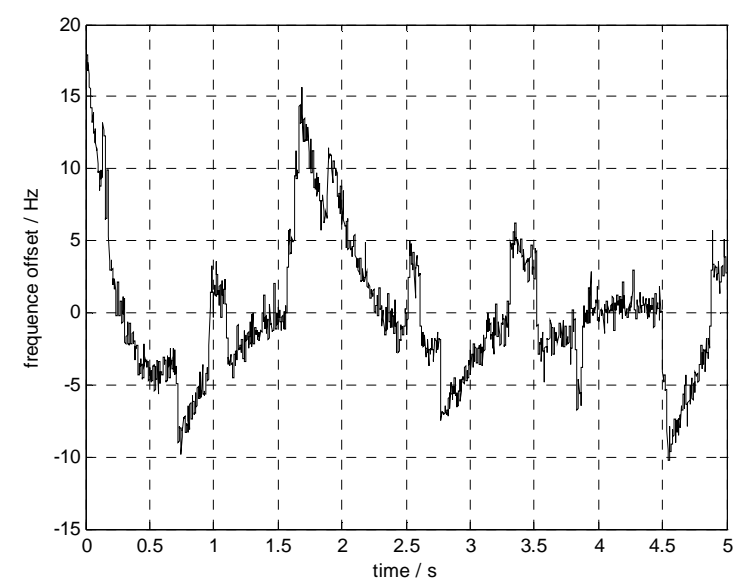

Figure 7. The performance of traditional FLL

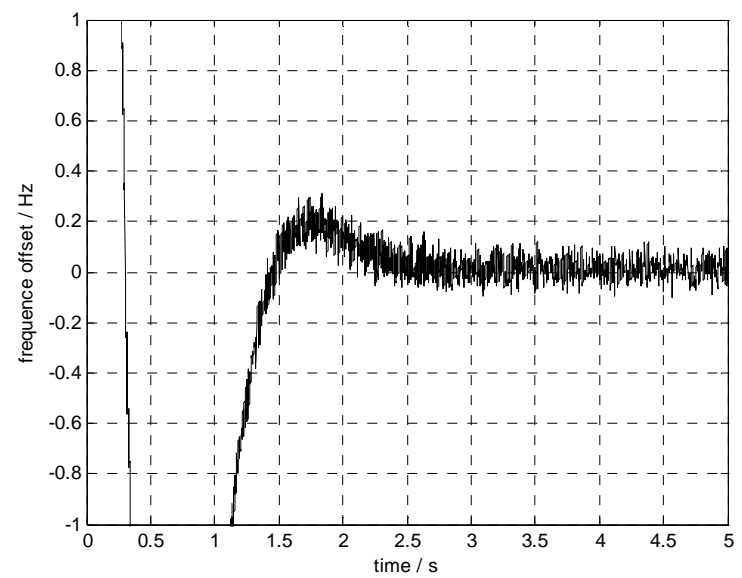

Figure 8. The performance of improved FLL

Fig. 7 shows that the traditional FLL cannot realize the stable and accurate tracking with the carrier to noise ratio at $25 \mathrm{dBHz}$. From Fig. 8 we can see that the improved FLL can realize stable tracking with the carrier processed by the SR processor and the tracking accuracy can reach $0.2 \mathrm{~Hz}$. This result verifies the significant effectiveness that the SR processor brings to FLL. Stochastic resonance has a great contribution to improve signal-to-noise ratio with transferring part noise energy to carrier energy. The effect of noise to FLL is greatly reduced and accurate tracking becomes a reality.

\section{Conclusion}

In this paper, we propose a SR processor which is used to weaken the effect of noise. Owing to its great improvement of SNR, our proposed FLL based on traditional FLL and SR processor can significantly improve the performance of carrier frequency synchronization, especially in low SNR condition. Simulation results indicates that the improved FLL has a high tracking accuracy with the carrier to noise ratio at $25 \mathrm{dBHz}$ and its final frequency steady-state offset is not more than $0.2 \mathrm{~Hz}$.

\section{ACKNOWLEDGMENT}

This work is supported by National Science Foundation of China(60972061,60972062 and 61032004), National High Technology Research and Development Program of China (“863”Program).

\section{REFERENCES}

[1] M. Han, Y. Wang, S. Wu and W. Cui, "A Study on Carrier Tracking FLL for Extremely-low-CNR Highly-dynamic Signals,”Modern Radar, vol. 31, pp.76-80 , 2009.

[2] K. Wiesenfeld, F. MOSS, "Stochastic resonance and the benefits of noise: from iceages to crayfish and SQUIDs”, Nature, vol. 373, pp. 33-36, 1995.

[3] Y. Leng, T. Wang, X. Qin, R. Li and Y. Guo, "Power spectrum research of twice samping stochastic resonance in a bistable system," ACTA PHYSICA SINICA, vol. 53, pp. 717-723, March 2004.

[4] R. Benzi, A. Sutera, and A. Vulpiani, "The mechanism of stochastic resonance”, J. Phys. A:Math. Gen, vol. 14, pp. L453-L457, 1981.

[5] G. Schmid, I. Goychuk, and P. Hanggi, "Stochastic resonance as a collective property of ion channel assemblies”, Europhysics Letters, vol. 56, pp.22-28, 2001.

[6] D. Kornack and P. Rakic, "Cell Proliferation without Neurogenesis in Adult Primate Neocortex,” Science, vol. 294, Dec. 2001, pp. 21272130, doi:10.1126/science.1065467.

[7] J. Douglass, L. Wilkens, E. Pantazelou, et al., "Noise enhancement of information transfer in crayfish mechanoreceptors by stochastic resonance”, Nature, vol. 365, pp. 337-340, 1993.

[8] V. Anishchenko, A. Neiman, and M. Safanova, "Stochastic resonance in chaotic systems”, Journal of Statistical Physics, vol. 70, pp. 183196, 1993.

[9] B. Xu, F. Duan, R. Bao, “Stochastic resonance with tuning system parameters: the application of bistable systems in signal processing”, Chaos, Solitons and Fractals, vol. 13, pp. 633-644, 2002

[10] F. Duan, B. Xu, "Parameter-induced stochastic resonance and baseband binary PAM signals transmission over an AWGN channel”, Int. J. Bifurcation and Chaos, vol. 13, pp. 411-415, 2003.

[11] Riter S. An Optimum Phase Reference Detector for Fully Modulated Phase Shift Keyed Signal [J]. IEEE Trans on Aerospace \& Electronics Systems, 1969.5 (4):627-631. 